\title{
Cerebral venous overdrainage: an under-recognized complication of cerebrospinal fluid diversion
}

\begin{abstract}
Kaveh Barami, MD, PhD
Department of Neurosurgery, Kaiser Permanente Northern California, Sacramento, California

Understanding the altered physiology following cerebrospinal fluid (CSF) diversion in the setting of adult hydrocephalus is important for optimizing patient care and avoiding complications. There is mounting evidence that the cerebral venous system plays a major role in intracranial pressure (ICP) dynamics especially when one takes into account the effects of postural changes, atmospheric pressure, and gravity on the craniospinal axis as a whole. An evolved mechanism acting at the cortical bridging veins, known as the "Starling resistor," prevents overdrainage of cranial venous blood with upright positioning. This protective mechanism can become nonfunctional after CSF diversion, which can result in posturerelated cerebral venous overdrainage through the cranial venous outflow tracts, leading to pathological states. This review article summarizes the relevant anatomical and physiological bases of the relationship between the craniospinal venous and CSF compartments and surveys complications that may be explained by the cerebral venous overdrainage phenomenon. It is hoped that this article adds a new dimension to our therapeutic methods, stimulates further research into this field, and ultimately improves our care of these patients.
\end{abstract}

http://thejns.org/doi/abs/10.3171/2016.6.FOCUS16172

KEY WORDS cerebrospinal fluid diversion; hydrocephalus; posture; shunt; Starling resistor; cerebral venous overdrainage

$\mathrm{T}$ HE intimate relationship between cerebral venous and cerebrospinal fluid (CSF) compartments is increasingly recognized as an important aspect of intracranial pressure (ICP) dynamics when one considers the craniospinal axis and the effects of gravity and atmospheric pressure on it as a whole..$^{5}$ From an evolutionary standpoint, as we became bipedal, certain accommodating mechanisms developed to prevent during upright positioning the overdrainage of fluid (CSF or blood) from the cranial to the spinal compartment, which could lead to intracranial hypotension. . $^{18,50,75,92,95}$

One such mechanism known as the "Starling resistor," which has been conserved across species, ${ }^{50}$ acts at the junction between cortical bridging veins that drain into the superior sagittal sinus, prevents siphoning of venous blood, and maintains ICP as we stand up. ${ }^{34,47,48}$ As the collapsible cortical bridging veins are draining into the rigid venous sinuses, they traverse the subarachnoid space and are subject to surrounding external CSF pressure. Essentially, the Starling resistor is a mechanism that maintains constant flow through collapsible tubes (such as cortical draining veins) when they are surrounded by variant ambi- ent pressure (CSF in the subarachnoid space) all contained in a rigid box, such as the skull in the hydraulic model shown in Fig. 1. The Starling resistor is a site of compression, also known as a "choke point," at the junction between the bridging vein and the sagittal sinus such that when pressure in the superior sagittal sinus drops during upright posture, the higher CSF pressure will choke the downstream connection to the sagittal sinus and prevent venous overdrainage. ${ }^{50}$ Concurrently pressure in the cerebral veins proximal to the Starling resistor is maintained at a higher level than CSF pressure because of the created back pressure. ${ }^{39,44}$ Thus the Starling resistor is responsible for maintaining the hierarchy of pressures of the various liquid compartments in the cerebrum, that is, arterial inflow pressure $>$ cerebral venous pressure $>$ subarachnoid CSF pressure $>$ sagittal sinus pressure. This, in turn, creates a pressure gradient to promote CSF drainage into the sagittal sinus. Various anatomical and MRI studies of the terminal regions of bridging veins are consistent with the idea that the Starling resistor functions as a flow resistor. $^{64,68,80,88}$

Several studies have shown that there are predictable 


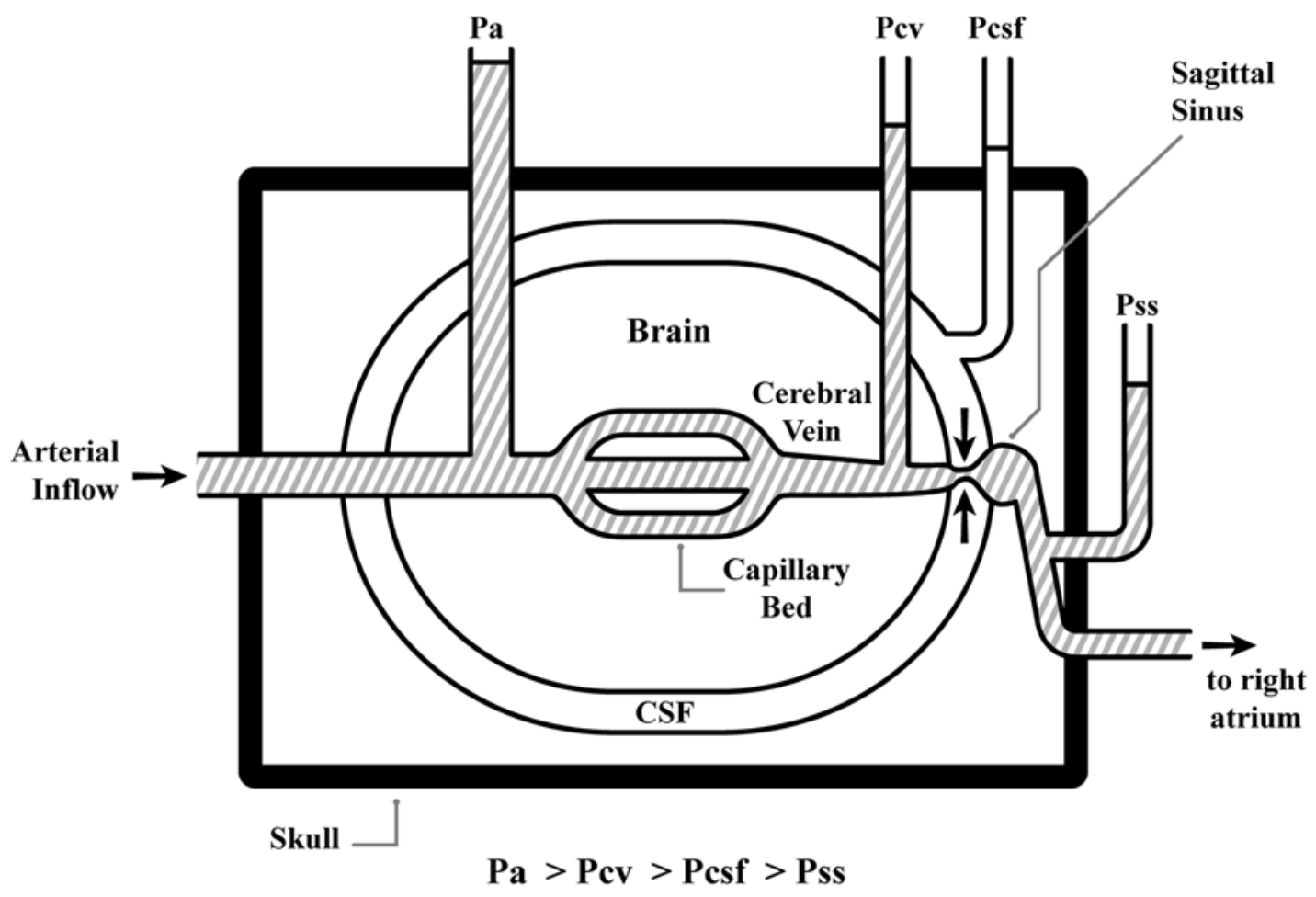

FIG. 1. Schematic of the hierarchy of pressures of various liquid components of the intracranial space. Arrows indicate the Starling resistor at the lateral lacunes (the junction between the cortical bridging vein and the sagittal sinus). Arterial inflow pressure (Pa) is higher than CSF pressure (Pcsf), which in turn is higher than pressure in the superior sagittal sinus (Pss). Pressure in the upstream cerebral veins (Pcv) is always maintained above Pcsf because of the constriction effect by the Starling resistor; this phenomenon also prevents venous overdrainage during upright positioning when sagittal sinus pressure falls. From Luce et al: J Appl Physiol 53:1496-1503, 1982. Published with permission.

changes in ICP and cerebral venous pressure with alterations in body posture. ${ }^{15,27}$ Intracranial pressure studies at different tilt angles have shown that there is a faster reduction of ICP at smaller angles and a slower reduction at higher angles. ${ }^{2,8,73}$ In healthy subjects, with upright posture there is a drop in ICP ranging between -5 and $5 \mathrm{~cm} \mathrm{H}_{2} \mathrm{O}$ with reference to the foramen magnum. The drop in ICP is related to the CSF loss from the cranial to the spinal compartment, usually about $3 \mathrm{ml}$, which is due to the differential in compliance of the 2 compartments. ${ }^{54}$ In contrast, in patients harboring shunts without antisiphon devices, pressures range from -15 to $-35 \mathrm{~cm} \mathrm{H}_{2} \mathrm{O}$ when they are upright. $15,41,45$ One method that allows us to quantify the cerebral venous pressure component of ICP during CSF diversion is Davson's steady-state formula, ${ }^{23} \mathrm{ICP}=\mathrm{Pcv}+$ $\left(\mathrm{I}_{f} \times \mathrm{R}_{\mathrm{o}}\right)$, where $P c v$ is cerebral venous pressure, $I_{f}$ is CSF formation rate (usually $0.3 \mathrm{ml} / \mathrm{min}$ ), and $R_{o}$ is CSF outflow resistance. One can see that if CSF is withdrawn at the same rate as its production and because $R_{o}$ approaches 0 , ICP will reflect cerebral venous pressure.

\section{Pathophysiology of Cerebral Venous Overdrainage}

The anatomy of the cranial venous outflow tracts is crucial in the pathophysiology of cerebral venous overdrainage. In the supine position the internal jugular veins are the main venous outflow pathways. ${ }^{28,82}$ During verticaliza- tion, because of atmospheric pressure, the internal jugular veins collapse and venous blood is rerouted to nonjugular pathways, specifically to the noncollapsible vertebral venous plexus. ${ }^{28,82}$ In a systematic ultrasonography and MRI analysis of the types and prevalence of human cerebral venous outflow, it was shown that there is predominantly jugular drainage in $72 \%$ of healthy volunteers. ${ }^{26}$ In $22 \%$ the jugular drainage equals the nonjugular drainage, and in $6 \%$ the drainage pattern is nonjugular. These findings suggest that in the general population there are variations in the anatomy of cranial venous outflow. ${ }^{7,82,94}$

Human cerebral venous outflow depends on central venous pressure and posture. ${ }^{26,36,63} \mathrm{~A}$ marked increase in central venous pressure, such as any Valsalva maneuver, completely reopens the internal jugular veins. ${ }^{26}$ In an ultrasonography study of the postural dependency of venous outflow, it was shown that blood flow in the internal jugular veins decreases from $700 \mathrm{ml} / \mathrm{min}$ in the supine position to $70 \mathrm{ml} / \mathrm{min}$ at $90^{\circ}$ elevation while flow in the vertebral veins is increased from 40 to $210 \mathrm{ml} / \mathrm{min} .{ }^{85}$ Total venous outflow declines from 740 to $280 \mathrm{ml} / \mathrm{min}$ from $0^{\circ}$ to $90^{\circ}$, with the largest decrease at $15^{\circ}$. One important finding is that flow in the vertebral veins exceeds internal jugular vein flow at $45^{\circ}$ of head elevation. ${ }^{85}$ The rigid spine seems to prevent epidural vein collapse, and negative epidural pressure may promote venous flow from the brain to the vertebral column. ${ }^{85}$

There is significant anatomical and functional conti- 
nuity between venous structures of the brain and spine. Analogous to the CSF craniospinal axis, the venous outflow network of the cranium and spine has been referred to as the "cerebrospinal venous system," which is further enhanced by the lack of venous valves. ${ }^{82}$ During postural changes this anastomosis serves an important function in pressure homeostasis of the cerebral venous system. ${ }^{82}$ The suboccipital cavernous sinus, situated in the deep muscular layers of the neck, serves as an important "relay station" anastamosing with the 2 main divisions of the craniospinal venous system (cranial and spinal) in the suboccipital region..$^{3,20}$ It is connected to the jugular bulb and internal jugular veins via the condylar veins and continues inferiorly into the deep cervical veins. ${ }^{3}$ The craniospinal venous system is essentially a large-capacity, valveless venous network in which flow is bidirectional and freely communicates with the sacral and pelvic veins. ${ }^{82}$ It has been shown that during Valsalva maneuvers, blood is squeezed out of the intraabdominal veins into the vertebral system and thus increasing intracranial pressure. ${ }^{7}$

The site where the craniospinal axis is atmospheric, referred to as the "zero point," is generally at the level of the foramen magnum. ${ }^{51-53}$ Magnaes has shown that in patients with shunts, during upright positioning the zero point is shifted caudally to the upper thoracic level; thus intracranial pressure can drop significantly relative to sagittal sinus pressures. ${ }^{6,51-53}$

In hydraulic terms, if the ambient CSF pressure in the subarachnoid space surrounding the bridging veins is low enough, it cannot constrict venous drainage into the sagittal sinus. One can see that when CSF diversion is accompanied by upright positioning, CSF pressure can drop below sagittal sinus pressure. It follows that low CSF pressure can render the Starling resistor nonfunctional and thus allowing overdrainage of cerebral venous blood through nonjugular pathways. ${ }^{5}$ This is consistent with the mathematical model proposed by Piechnik et al. in which CSF and sagittal sinus pressures become dissociated.$^{70}$ This could lead to exacerbation of intracranial hypotension ${ }^{76}$ and, depending on varying circumstances (such as head elevation, position, individual anatomy, and compensatory mechanisms), congestion in veins such as the suboccipital cavernous sinus that connects the jugular and nonjugular pathways and accommodates the diverted venous volume. ${ }^{5}$ A horizontal position would increase ICP with respect to the dural sinus pressure, in other words, reestablishing the normal hierarchical relationships in the pressures and thus terminating the cascade of intracranial hypotension. ${ }^{5}$

The phenomenon of venous overdrainage from the cranium was first described in 2000 by Barami et al. ${ }^{6}$ They measured ICP and compliance in patients with chronic shunts that had been externalized. The data allowed for calculating whether displacements in CSF volume accounted for posture-related changes in ICP. A key finding was that in these patients with chronic shunts, the almost instantaneous drop in ICP in the upright position occurred even when the shunt was externalized and not allowed to drain. The data showed that for the pressure volume indices measured, the amount of fluid shift to explain pressure changes exceeded the amount of intracranial CSF. Therefore, it was hypothesized that the other liquid component of the cranial cavity, that is, venous blood, was responsible for the drop in ICP referred to as "venous overdrainage."

\section{Disease Entities Implicating Cerebral Venous Overdrainage}

A survey of the relevant literature revealed several categories of complications that might be explained by cerebral venous overdrainage: 1) venous congestion or engorgement in the spine causing mass effect, 2) suboccipital venous congestion causing remote site intracranial hemorrhage, 3) low pressure hydrocephalus, and 4) ventriculomegaly associated with craniectomy (Fig. 2).

\section{Venous Congestion-Associated Cervical Myelopathy After CSF Diversion: Miyazaki Syndrome}

Cervical myelopathy associated with intracranial hypotension after ventriculoperitoneal shunting was first described by Miyazaki in 1998. ${ }^{59}$ Subsequently in 2002 Matsumoto presented a case of cervical radiculomyelopathy in a 69-year-old man with a ventriculoperitoneal shunt that had been placed 2 years earlier and in whom imaging showed an enhanced "extramedullary mass" causing cord compression. ${ }^{57}$ The patient's symptoms resolved after removal of the shunt. Imaging findings and symptoms were thought to represent engorged epidural veins caused by intermittent intracranial hypotension due to postural changes of CSF pressure. In 2009 Martínez-Lage et al. presented the case of a 20 -year-old woman with a ventriculoperitoneal shunt that had been placed 14 years earlier and who had an incidental finding of an engorged cervical epidural venous plexus. ${ }^{56}$ In a review of the literature on similar cases spanning patient ages from the late teens to the geriatric years and all harboring shunts, these authors documented an interval from 22 months to 27 years between CSF shunt placement and the appearance of symptoms. Ulrich et al. have performed a more current literature review of chronic overshunting-associated venous congestion causing myelopathy and also described a patient with congenital cervical canal stenosis who had presented with cervical myelopathy and dilated venous plexus at the craniocervical junction.${ }^{84}$ Symptoms and imaging findings in that case resolved after shunt revision and the addition of an antisiphon device leading to a gradual increase in ICP. The preferential shunting of blood through the vertebral plexus has been angiographically demonstrated in another case described by Wolfe et al., who found the collapse of both internal jugular veins and engorged muscular and pericondylar veins shunting blood into the epidural venous plexus after ventriculoperitoneal shunting. ${ }^{91}$

These cases demonstrate that in patients with CSF diversion, there could be increased venous pooling in the spinal compartment whereby a subset of the patients become symptomatic. ${ }^{17}$ The dominant hypothesis among the cases reported so far has been that, according to the Monro-Kellie doctrine, ${ }^{19}$ given the loss of CSF there should be an increase in blood flow as a compensatory mechanism $^{30,60,61}$ where selective uptake of contrast material during MRI occurs in the pachymeninges, as compared with that in the leptomeninges, due to the lack of a blood-brain barrier. Biopsy of the meninges in patients with intracra- 


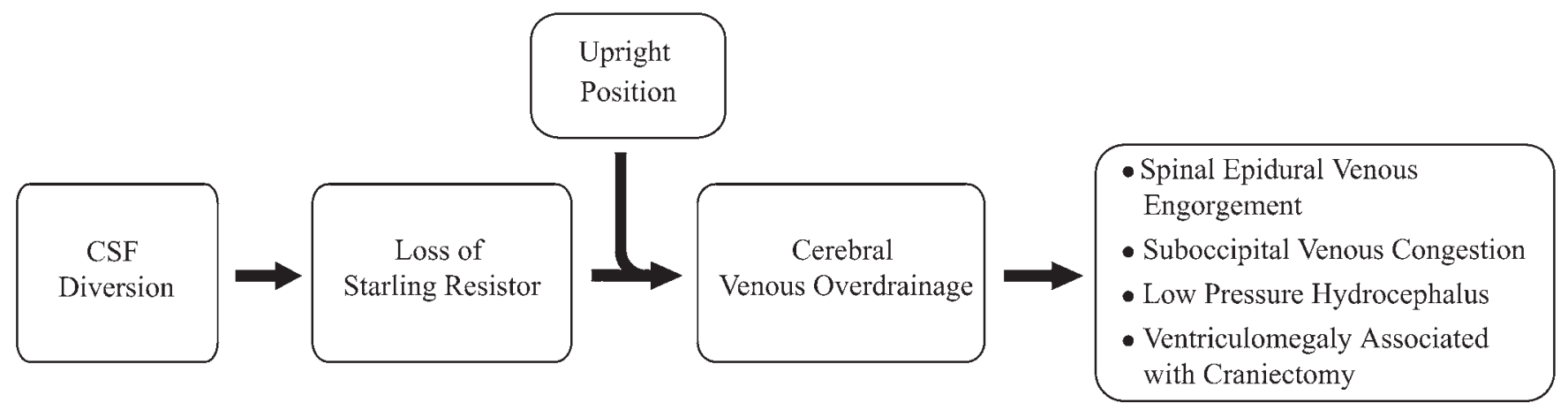

FIG. 2. Cerebral venous overdrainage and related complications may occur during CSF diversion with upright positioning due to loss of the Starling resistor effect on cortical bridging veins. Adapted from Childs Nerv Syst, The cerebral venous system and the postural regulation of intracranial pressure: implications in the management of patients with cerebrospinal fluid diversion, Vol 32 , 2016, pp 599-607, Barami K, Sood S. With permission of Springer.

nial hypotension suggests that a thin subdural zone of fibroblasts, fibrocollagenous proliferation, and small, thinwalled dilated blood vessels form the anatomical basis of pachymeningeal enhancement suggestive of a reactive secondary phenomenon. ${ }^{62}$ Interestingly, Caruso et al. have also described the same engorgement of cervical epidural veins causing symptoms after craniectomy that resolved after cranioplasty. ${ }^{14}$ Their case report highlights the fact that perhaps it is the increased compliance and loss of CSF outflow resistance associated with craniectomy that leads to the loss of the Starling resistor effect and thus the cerebral venous overdrainage via the vertebral venous plexus causing cervical epidural vein engorgement. Cranioplasty, in turn, restores compliance and CSF outflow resistance and resolves symptoms.

Several items argue against the explanation that there is venous hyperemia to compensate for CSF loss according to the Monro-Kellie doctrine. First, there is angiographic evidence during intracranial hypotension of a collapse of internal jugular veins and engorgement of venous structures such as muscular branches and pericondylar veins. This suggests selective shunting of venous blood into the vertebral venous plexus. ${ }^{91}$ Second, there is evidence that after CSF diversion from lumbar puncture, flow in the venous sinuses actually drops and is not increased..$^{13}$ Third, in other situations in which there is significant loss of $\mathrm{CSF}^{71}$ or another component of the cranial cavity, such as brain tissue loss after hemispherectomy, there has been no demonstrated compensatory increase in cerebral venous volume. Fourth and last, as stated earlier, the same pattern of spinal epidural engorgement occurs in cases without CSF loss but in which brain compliance increases and CSF outflow resistance decreases ${ }^{14}$ conditions that would promote cerebral venous overdrainage.

The phenomenon of cervical venous engorgement after CSF diversion seems to be more congruent with cerebral venous overdrainage when it is due to CSF loss, the Starling resistor at the bridging veins becomes nonfunctional, and there is cerebral venous overdrainage with preferential flow and engorgement in the vertebral venous plexus due to postural changes.

\section{Remote Site Intracranial Hemorrhage}

Remote site intracranial hemorrhage is a rare compli- cation following intracranial surgery and spinal procedures with a reported overall incidence of $0.08 \%$ to $0.6 \%$ after supratentorial craniotomies. $4,10,12,32,33,35,55,86$ Its etiology remains controversial; therefore recommendations on avoiding it are difficult to outline. Reports of infratentorial bleeds following supratentorial surgery and vice versa have been recently reviewed by Garg et al. ${ }^{35}$ The most common hemorrhage pattern is cerebellar hemorrhage following supratentorial surgery. ${ }^{4}$ Specifically blood is seen in the sulci of one or both cerebellar hemispheres and vermis facing the tentorium in an alternating pattern of hyperdense (blood) and hypodense (cerebellum) stripes referred to as the "zebra sign." 32 This complication was first reported by Yaşargil and Yonekawa. ${ }^{93}$ Remote site intracranial bleeds can also occur less frequently after endonasal surgery, ${ }^{46}$ bur hole evacuation of subdural hematomas ${ }^{89}$ and spinal surgery at all levels that are associated with CSF loss. ${ }^{32}$

With regard to adult hydrocephalus, remote intracranial bleeds (most commonly cerebellar hemorrhages) have also been seen following lumboperitoneal shunting ${ }^{83}$ simple lumbar puncture, ${ }^{9}$ reduction in the valve pressure of a ventriculoperitoneal shunt, ${ }^{69}$ multiple manual pumping of a ventriculoperitoneal shunt valve, ${ }^{42}$ and placement of an external ventricular drain. ${ }^{22}$ All seem to have CSF loss as a common denominator. Although the etiology remains controversial, several aspects of this complication seem to be agreed on: 1) involvement of CSF drainage, 2) hemorrhage of venous origin rather than arterial, 3) location of a bleed typically subarachnoid with a variable intraparenchymal element, and 4) predilection for superior vermian vein territory, that is, superior cerebellar surface and vermis. ${ }^{4,10,33,35}$ In general the incidence of severe neurological deficits is $9.7 \%$ and associated morbidity is $14.5 \% .^{35}$ Moreover 54\% of the patients develop symptoms 10 hours postoperatively, and the most common presenting symp-

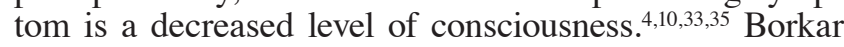
et al. have recently performed a literature review on this complication. ${ }^{10}$ One interesting finding is that supratentorial bleeds after infratentorial surgeries are uncommon as compared with the converse, and nearly all reported cases of the former had been performed in the sitting position, suggesting that postural factors seem to be involved. . $10,33,35^{-1}$

The most common pathophysiological explanation for 
this complication has been cerebellar "sag" due to CSF hypovolemia causing transient occlusion of posterior fossa bridging veins and secondary venous infarction. With regard to the cerebellar sag hypothesis, other authors have discussed the possibility of caudal migration of the cerebellum, which in turn causes stretching, kinking, or shearing of the superior vermian veins.,11,33 Another mechanism proposed by Seoane and Rhoton is excessive rotation and extension of the head during pterional craniotomies, causing internal jugular vein compression by the transverse process of the atlas. ${ }^{77}$ This, in turn, occludes venous outflow with a resultant increase in intraluminal pressure in the veins, ${ }^{77}$ yet it seems an unlikely mechanism in cases of spinal procedures in which generally the head is kept neutral.

The above complications could be explained from the vantage point of cerebral venous overdrainage. The Starling resistor becomes nonfunctional during significant CSF loss. Should the loss of CSF occur in the supine position, cerebral venous pressures are low and there is slow flow in the outflow veins, which has been demonstrated by Canhão et al., as mentioned previously. ${ }^{13}$ On the other hand, if the loss of CSF is accompanied by head elevation without compensatory mechanisms to increase central venous pressure, there will be ensuing cerebral venous overdrainage from the noncollapsible major sinuses into the outflow veins with preferential congestion in the suboccipital cavernous sinus, ${ }^{29}$ the "relay station" between the cranial and spinal components of the craniospinal venous system as the venous outflow is redirected from the internal jugular veins to the vertebral venous plexus.

In summary, the cerebral venous overdrainage hypothesis may explain the phenomenon of remote cerebellar bleeds after rapid and/or excessive CSF loss associated with CSF diversion. During the loss of CSF, there is dissociation of CSF and cerebral venous pressures ${ }^{70}$ and the Starling resistor becomes nonfunctional. Compensatory mechanisms may be lacking, and depending on the degree of head elevation, individual variations in anatomy, including cranial venous outflow, there could be venous pooling in the posterior fossa veins causing hemorrhages. This may also explain why remote site hemorrhages are rare in the pediatric age group since their drops in CSF pressures are not as significant as those in adults during CSF loss given their smaller stature. ${ }^{37,43}$

\section{Low Pressure Hydrocephalus}

Low pressure hydrocephalus is loosely defined as a paradoxical increase in the size of ventricles despite low to normal CSF pressures in patients with CSF diversion and is associated with neurological deterioration. Symptoms include postural headaches, lethargy, obtundation, and cranial neuropathies. Most cases involve patients with shunted hydrocephalus, whereas others involve patients with external ventricular drains as well as those with CSF rhinorrhea. The first case series on low pressure hydrocephalus was performed by Pang and Altschuler in 1994. ${ }^{67}$ They reported on 12 patients who had previously tolerated shunts but later presented with ventriculomegaly and low CSF pressures. All patients except one were treated with external ventricular drainage at subatmospheric pres- sures, which were slowly increased, and new shunts were finally reinserted. No correlation was found between ICP and ventricular size, and improvement in symptoms correlated with a decrease in the size of ventricles. The average calculated pressure volume index in 3 patients was $43.9 \pm 4.6$ (190\% of predicted normal value). The authors proposed that the development of low pressure hydrocephalus is related to changes in the viscoelastic modulus of the brain due to the expulsion of extracellular water from the brain and due to structural changes caused by chronic overstretching. They asserted that susceptible patients are those who, due to bioatrophic changes, have innate low brain elasticity and that the goal of treatment is to allow the entry of water into the brain parenchyma to restore brain viscoelasticity. ${ }^{67}$ Subsequently other authors have argued against the viscoelastic hypothesis and the subatmospheric method of treatment. Vassilyadi et al. described 2 cases of low pressure hydrocephalus that occurred hours to days after spinal cystopleural shunting in patients who had previously had shunts. ${ }^{87}$ These authors argued that the timing of the development of low pressure hydrocephalus did not correspond to that required for viscoelastic changes and that the transmantle pressure gradient causing excessively low convexity subarachnoid pressures also caused the ventriculomegaly. One patient improved with positive end-expiratory pressures and clamping of the cystopleural shunt; the second patient was treated by changing to a flow-regulated valve. These authors also noted that their 2 patients were similar to 2 of Pang and Altschuler's patients who developed low pressure hydrocephalus immediately after lumbar puncture. Clarke et al. described 2 patients with low pressure hydrocephalus who were treated with the subatmospheric method and whose symptoms improved during negative pressure drainage; however, despite the placement of ventriculoatrial shunts and the normalization of ventricular size, outcomes were poor. ${ }^{16}$ Another report on low pressure hydrocephalus in patients with shunts illustrates that subatmospheric drainage may be unnecessary and argues against any surgical intervention in the treatment of such patients. ${ }^{25}$ In this article, Dias et al. described 2 cases of low pressure hydrocephalus in patients with shunts who had presented with postural symptoms precipitated by lumbar puncture; interestingly both cases resolved, and ventricle size became normal with only enforced recumbency without the need for shunt revision. These authors advised against shunt revision in cases of low pressure hydrocephalus. It is possible that simple bed rest allows for the reestablishment of the normal CSF-venous system interaction.

Other authors have supported the subatmospheric method for the treatment of low pressure hydrocephalus. Daniel et al. documented a case of low pressure hydrocephalus that developed after CSF rhinorrhea following hemispherectomy; the patient was successfully treated with subatmospheric drainage. ${ }^{21}$ Owler et al. demonstrated that 4 of their 5 low pressure hydrocephalus cases improved with subatmospheric drainage and conversion to low-pressure or valveless shunts, ${ }^{66}$ although they argued against Pang and Altschuler's hypothesis ${ }^{67}$ on the loss of water from the parenchyma since ventriculomegaly during low pressure hydrocephalus was associated with con- 
tinued transependymal passage of CSF with an increase in brain extracellular fluid.

There is ongoing debate regarding the genesis of low pressure hydrocephalus. Akins et al. suggested, based on a poroelastic model, that the brain acts as a "sponge" and that due to increased brain permeability, extracellular fluid increases, leading to the flux of fluid in and out of the brain as well as an increased ventricular volume. ${ }^{1}$ Lastly, Lesniak et al. attempted to explain the paradoxical ventriculomegaly seen in patients with low pressure hydrocephalus by suggesting that in the acute phase of hydrocephalus, acute distension of the ventricular system increases compliance of the cranial vault, thus shifting the pressure-volume curve to the right. ${ }^{49}$

The above reports show that there are clearly a group of patients with CSF diversion who develop low pressure hydrocephalus, respond well to CSF drainage at subatmospheric pressures, and are gradually transitioned to positive pressures. As regards the etiology and treatment of this condition, the cerebral venous overdrainage hypothesis offers several insights: CSF diversion causes CSF hypotension leading to the loss of the Starling effect on draining cortical veins. Interestingly in an MRI study of intracranial CSF volume after lumbar puncture, it was shown that most of the CSF loss occurred from the cortical sulci, suggesting a loss of pressure in the cortical subarachnoid space. ${ }^{38}$ Upright positioning (aggravated by the loss of compensatory mechanisms) leads to cerebral venous overdrainage and thus a parallel decrease in cerebral venous pressures. This derangement of the normal hierarchy of cerebral pressures initiates a vicious cycle of intracranial hypotension resolved by reestablishing the normal hierarchy. Ventriculomegaly could be explained by cerebral venous hypotension and low pressures in the superior sagittal sinus and similarly in the deep cerebral veins and periventricular veins (which are contiguous with the sagittal sinus and also lack Starling resistors), leading to an increased pressure gradient and CSF flow from ventricle to parenchyma and ventriculomegaly. ${ }^{58,72}$ This also causes transependymal CSF flow leading to periventricular edema, as suggested by Portnoy et al. ${ }^{72}$ Bed rest (during external ventricular drainage) allows compensatory mechanisms to act (increase in central venous pressure), reducing this pressure gradient. Moreover ventricular drainage contributes to normalization of the CSF to venous pressure gradient and reestablishment of the normal CSF-venous system relationship. The significance of posture to ventricular size was demonstrated by McCullough et al. in $1986 .{ }^{58}$ In their report 5 patients with shunted hydrocephalus and harboring antisiphon devices developed postural symptoms and enlargement of the ventricles when they were upright; the ventriculomegaly and symptoms resolved after horizontal positioning. These authors also noted that all of the patients were relatively tall. The successful treatment of low pressure hydrocephalus cases with positive end-expiratory pressure $^{87}$ and recumbency ${ }^{25}$ are also consistent with the fact that posture-related hydrostatic effects play a role in the pathophysiology of this disease entity.

More recently Hamilton and Price have reported their experience with inappropriately low pressure acute hydrocephalus and have provided insight into its treatment. ${ }^{40} \mathrm{In}$ their series all patients had anatomical obstruction of CSF flow into the subarachnoid space. They concluded that, in addition to forced drainage of the ventricles, treatment should be directed at restoring communication between the cortical subarachnoid space and the ventricular system by endoscopic third ventriculostomy and by increasing dural sinus pressures via a neck tourniquet and/or abdominal binder. From the vantage point of cerebral venous overdrainage, the application of a neck tourniquet and abdominal binder restricts cranial venous outflow and promotes blood flow into the vertebral venous system, respectively. Endoscopic third ventriculostomy diverts CSF out of the ventricles into the subarachnoid space, effectively reducing the ventricle to periventricular tissue gradient and increasing subarachnoid CSF pressures. Taken as a whole, these modalities restore the hierarchy of pressures as well as conditions for a functioning Starling resistor and thus reduce ventricular size and normalize intracranial pressure dynamics.

Analysis of the above articles reveals several other interesting observations. Pressure volume index is a measure of neural axis compliance with a normal value of $25.9 \pm 3.7$ in adults and a range from 8.2 to 30.1 in the pediatric population..$^{79}$ Increased brain compliance indices calculated by Pang and Altschuler ${ }^{67}$ are almost identical to those in the Barami et al. report ${ }^{6}(43.9 \pm 4.6$ vs $42.8 \pm$ $4.5)$, yet ventricle sizes in the latter study's cohort were mostly small and were large in the former study. This suggests that increased brain compliance can occur in patients with shunts without ventriculomegaly, and as suggested by Magnaes, it is the loss of CSF that causes an increase in compliance and not necessarily enlargement of the ventricles. ${ }^{6,52}$ The term "subatmospheric" may be a misnomer if one considers Magnaes' findings in which in patients with shunts there was caudal displacement of the zero (atmospheric) point; ${ }^{51-53}$ thus the subatmospheric method of lowering the external ventricular drain below the external auditory meatus might have been a matching of the drip chamber to the lowered zero point. Interestingly several patients in previous series developed low pressure hydrocephalus hours or days after CSF diversion, which makes it unlikely that chronic inflammatory and atrophic changes (as suggested by Pang and Altschuler ${ }^{67}$ ) have taken place. Lastly, some of the symptoms described in Pang and Altschuler's series, specifically gait ataxia and hypertonicity, could be explained by cervical myelopathy associated with cerebral venous overdrainage (Miyazaki syndrome), as mentioned earlier.

\section{Ventriculomegaly Associated With Craniectomy}

Another condition in which the normal hierarchy of pressures is deranged follows the removal of a large portion of the skull known as "craniectomy," which is performed as a life-saving maneuver during malignant intracranial hypertension. ${ }^{81}$ In a study in cats Shapiro et al. showed that when a portion of the skull is removed, the compliance (pressure volume index) of the brain increases and the CSF outflow resistance decreases; $; 8,79$ therefore, according to the Davson formula above, ${ }^{23}$ since CSF outflow resistance approaches 0, again ICP becomes dependent on cerebral venous pressure ${ }^{67}$ Moreover, dural open- 
ing after craniectomy further increases brain compliance and decreases CSF outflow resistance. ${ }^{78}$ In Shapiro et al.'s study the contribution of $\left(\mathrm{I}_{f} \times \mathrm{R}_{\mathrm{o}}\right)$ went from $22 \%$ in the intact cat to $13 \%$ after craniectomy and to $6 \%$ after dural opening. ${ }^{78}$ Fodstad et al. ${ }^{31}$ and Osterholm ${ }^{65}$ have shown that in humans superior sagittal sinus pressure drops after craniectomy and rises after cranioplasty. In the report by Fodstad and colleagues, typical symptoms in patients who had undergone craniectomy, grouped as "syndrome of the trephined," such as headache, vertigo, tinnitus, extremity paresis, and dysphasia, were aggravated by body position and Valsalva maneuvers and improved dramatically after cranioplasty; thus it was suggested that these symptoms were caused by CSF hydrodynamic changes related to craniectomy. ${ }^{31}$ Fodstad et al. also commented on the effect of atmospheric pressure acting directly on cerebral tissue during craniectomy. According to them, during an upright position intracranial pressure would equalize with the atmospheric pressure. ${ }^{31}$

A well-known complication after craniectomy is ventriculomegaly. ${ }^{81}$ In 2007 Waziri et al. reported an 88\% incidence of communicating hydrocephalus in their cohort of 17 patents who had undergone hemicraniectomy, half of whom required ventriculoperitoneal shunts. ${ }^{90}$ The study excluded patients who had undergone hemicraniectomy for subarachnoid hemorrhage, intraventricular hemorrhage, or infection, that is, confounding variables that may have contributed independently to hydrocephalus. The mean time to develop hydrocephalus was 7.7 days. These authors concluded that hydrocephalus and enlarging extraaxial collections over the convexities were universal findings in hemicraniectomy patients and that this suggested an imbalance between CSF production and drainage into the venous sinuses caused by disruption of pulsatile ICP dynamics after craniectomy. Therefore, early cranioplasty was recommended to restore normal ICP dynamics. ${ }^{90}$ Rahme et al. reported on their experience with 17 patients who had undergone craniectomy. ${ }^{74}$ In contrast to Waziri et al.'s findings, Rahme and colleagues found that none of the patients developed clinically significant hydrocephalus requiring shunting. They concluded that the discrepancy between the 2 studies lay in the definition of hydrocephalus and indications for shunting, that is, the distinction between symptomatic hydrocephalus versus asymptomatic ventriculomegaly.

One study that may explain this discrepancy was performed by De Bonis et al., ${ }^{24}$ who reported their experience with posttraumatic hydrocephalus in patients who had undergone decompressive hemicraniectomy after traumatic brain injury. They analyzed the area of craniotomy and the distance of the craniotomy from the midline. Results showed that patients who had undergone craniectomies with a superior limit less than $25 \mathrm{~mm}$ from the midline had a markedly increased risk of developing hydrocephalus. They hypothesized, based on the Starling resistor concept, that during each cardiac cycle in which extracellular fluid is produced during systole and absorbed in diastole, an imbalance between the production and absorption of extracellular fluid in favor of absorption would cause a decrease in brain parenchyma volume and consequently increase the size of the ventricles. ${ }^{24}$ Thus in patients with craniectomies too close to the midline, the loss of CSF pressure surrounding the cortical draining veins in diastole would cause an increase in venous outflow. In turn, cerebral venous overdrainage would cause an increase in extracellular fluid absorption, leading to a loss of brain parenchyma volume and ventriculomegaly. In light of their observations, this group also attempted to reconcile the disparate findings of Waziri et al. and Rahme et al. by noting that in the former study, the superior margin of craniectomies was within $1-2 \mathrm{~cm}$ of the sagittal sinus, which led to a high incidence of hydrocephalus. On the other hand, Rahme et al. most likely had no cases of hydrocephalus since the superior margin of the craniectomies was greater than $25 \mathrm{~mm}$ off the midline. ${ }^{24}$ If one considers Fodstad et al.'s remarks about the effects of atmospheric pressure in patients with skull defects, ${ }^{31}$ it is also conceivable that in patients with craniectomies too close to the midline, the sagittal sinus is more prone to collapse by atmospheric pressure. In this situation the normal CSF to sagittal sinus pressure gradient would be lost, leading to poor CSF absorption and thus an increase in extraaxial fluid collections and ventriculomegaly; cranioplasty would reverse this effect. $24,31,65,74,81,90$ The same principles may be applicable to Caruso et al.'s findings that engorgement of cervical epidural veins causing symptoms occurred after craniectomy and resolved after cranioplasty, ${ }^{14}$ suggesting that venous overdrainage could occur in craniectomy patients in whom cranial compliance increases and CSF outflow resistance drops.

\section{Therapeutic Implications and Future Directions}

When one considers the coupling between cerebral venous and CSF compartments of the craniospinal axis in the management of adult hydrocephalus, it may be important to avoid cerebral venous overdrainage in vulnerable patients. Such patients include those harboring CSF shunts whose CSF drainage equals or exceeds the CSF formation rate (approximately $0.3 \mathrm{ml} / \mathrm{min}$ ) as well as craniectomy patients in whom cranial compliance is increased. Regarding patterns of cerebral venous outflow, it seems that the highest-risk patients are those with a predominant nonjugular pathway of drainage. In these high-risk patients the transition from a supine to an upright position should occur in a controlled fashion. Patients with low pressure hydrocephalus should be treated with a trial of supine positioning, the prevention of further CSF loss (including diagnostic lumbar puncture), and the restoration of the normal hierarchy of pressures of the fluid compartments of the craniospinal axis. Assessment via brain and spinal MRI with contrast to demonstrate cerebral venous overdrainage and Doppler ultrasonography to assess flow through the cranial venous outflow tracts are also helpful noninvasive adjuncts in the management of adult hydrocephalus and may guide therapy. Antisiphon devices may mitigate the long-term effect of chronic CSF overdrainage from a shunt causing cerebral venous overdrainage. Further research must be done to better define patients at risk and to identify modulating factors of the cerebral venous overdrainage phenomenon. 


\section{Acknowledgments}

I thank Bahar Hanjani for the illustration in Fig. 2.

\section{References}

1. Akins PT, Guppy KH, Axelrod YV, Chakrabarti I, Silverthorn J, Williams AR: The genesis of low pressure hydrocephalus. Neurocrit Care 15:461-468, 2011

2. Andresen M, Juhler M: Intracranial pressure following complete removal of a small demarcated brain tumor: a model for normal intracranial pressure in humans. J Neurosurg 121:797-801, 2014

3. Arnautović KI, al-Mefty O, Pait TG, Krisht AF, Husain MM: The suboccipital cavernous sinus. J Neurosurg 86:252-262, 1997

4. Baeesa SS: Remote cerebellar hemorrhage in neurosurgery. Neurosciences (Riyadh) 17:305-308, 2012

5. Barami K, Sood S: The cerebral venous system and the postural regulation of intracranial pressure: implications in the management of patients with cerebrospinal fluid diversion. Childs Nerv Syst 32:599-607, 2016

6. Barami K, Sood S, Ham SD, Canady AI: Postural changes in intracranial pressure in chronically shunted patients. Pediatr Neurosurg 33:64-69, 2000

7. Batson OV: The function of the vertebral veins and their role in the spread of metastases. 1940. Clin Orthop Relat Res (312):4-9, 1995

8. Bergsneider M, Yang I, Hu X, McArthur DL, Cook SW, Boscardin WJ: Relationship between valve opening pressure, body position, and intracranial pressure in normal pressure hydrocephalus: paradigm for selection of programmable valve pressure setting. Neurosurgery 55:851-859, 2004

9. Bernal-García LM, Cabezudo-Artero JM, Ortega-Martínez M, Fernández-Portales I, Ugarriza-Echebarrieta LF, Pineda-Palomo M, et al: [Remote cerebellar hemorrhage after lumbar spinal fluid drainage. Report of two cases and literature review.] Neurocirugia (Astur) 19:440-445, 2008 (Span)

10. Borkar SA, Lakshmiprasad G, Sharma BS, Mahapatra AK: Remote site intracranial haemorrhage: a clinical series of five patients with review of literature. Br J Neurosurg 27:735738,2013

11. Brisman MH, Bederson JB, Sen CN, Germano IM, Moore F, Post KD: Intracerebral hemorrhage occurring remote from the craniotomy site. Neurosurgery 39:1114-1122, 1996

12. Brockmann MA, Groden C: Remote cerebellar hemorrhage: a review. Cerebellum 5:64-68, 2006

13. Canhão P, Batista $P$, Falcão F: Lumbar puncture and dural sinus thrombosis - a causal or casual association? Cerebrovasc Dis 19:53-56, 2005

14. Caruso RD, Smith MV, Chang JK, Wasenko JJ, Rosenbaum AE: Giant cervical epidural veins after craniectomy for head trauma. AJNR Am J Neuroradiol 19:903-906, 1998

15. Chapman PH, Cosman ER, Arnold MA: The relationship between ventricular fluid pressure and body position in normal subjects and subjects with shunts: a telemetric study. Neurosurgery 26:181-189, 1990

16. Clarke MJ, Maher CO, Nothdurft G, Meyer F: Very low pressure hydrocephalus. Report of two cases. J Neurosurg 105:475-478, 2006

17. Clarot F, Callonnec F, Douvrin F, Hannequin D, Simonet J, Proust B, et al: Giant cervical epidural veins after lumbar puncture in a case of intracranial hypotension. AJNR Am J Neuroradiol 21:787-789, 2000

18. Conroy GC, Vannier MW, Tobias PV: Endocranial features of Australopithecus africanus revealed by 2- and 3-D computed tomography. Science 247:838-841, 1990

19. Cushing HW: The third circulation, in Studies in Intracranial Studies in Physiology and Surgery. London: Oxford University Press, 1926, pp 1-51
20. Dagain A, Vignes JR, Dulou R, Dutertre G, Delmas JM, Guerin J, et al: Junction between the great cerebral vein and the straight sinus: an anatomical, immunohistochemical, and ultrastructural study on 25 human brain cadaveric dissections. Clin Anat 21:389-397, 2008

21. Daniel RT, Lee GY, Halcrow SJ: Low-pressure hydrocephalic state complicating hemispherectomy: a case report. Epilepsia 43:563-565, 2002

22. Das KK, Nair P, Mehrotra A, Sardhara J, Sahu RN, Jaiswal AK, et al: Remote cerebellar hemorrhage: Report of 2 cases and review of literature. Asian J Neurosurg 9:161-164, 2014

23. Davson H: Formation and drainage of the cerebrospinal fluid in Shapiro K, Marmarou A, Portnoy H (eds): Hydrocephalus. New York: Raven Press, 1984, pp 3-40

24. De Bonis P, Pompucci A, Mangiola A, Rigante L, Anile C: Post-traumatic hydrocephalus after decompressive craniectomy: an underestimated risk factor. J Neurotrauma 27:19651970, 2010

25. Dias MS, Li V, Pollina J: Low-pressure shunt 'malfunction' following lumbar puncture in children with shunted obstructive hydrocephalus. Pediatr Neurosurg 30:146-150, 1999

26. Doepp F, Schreiber SJ, von Münster T, Rademacher J, Klingebiel R, Valdueza JM: How does the blood leave the brain? A systematic ultrasound analysis of cerebral venous drainage patterns. Neuroradiology 46:565-570, 2004

27. Drake JM, Tenti G, Sivalsganathan S: Computer modeling of siphoning for CSF shunt design evaluation. Pediatr Neurosurg 21:6-15, 1994

28. Epstein HM, Linde HW, Crampton AR, Ciric IS, Eckenhoff JE: The vertebral venous plexus as a major cerebral venous outflow tract. Anesthesiology 32:332-337, 1970

29. Evins AI, Boeris D, Burrell JC, Ducati A: Postoperative intracranial hypotension-associated venous congestion: case report and literature review. Clin Neurol Neurosurg 115:2243-2246, 2013

30. Fishman RA, Dillon WP: Dural enhancement and cerebral displacement secondary to intracranial hypotension. Neurology 43:609-611, 1993

31. Fodstad H, Love JA, Ekstedt J, Fridén H, Liliequist B: Effect of cranioplasty on cerebrospinal fluid hydrodynamics in patients with the syndrome of the trephined. Acta Neurochir (Wien) 70:21-30, 1984

32. Friedman JA, Ecker RD, Piepgras DG, Duke DA: Cerebellar hemorrhage after spinal surgery: report of two cases and literature review. Neurosurgery 50:1361-1364, 2002

33. Friedman JA, Piepgras DG, Duke DA, McClelland RL, Bechtle PS, Maher CO, et al: Remote cerebellar hemorrhage after supratentorial surgery. Neurosurgery 49:1327-1340, 2001

34. Fry D, Thomas L, Greenfield J: Flow in collapsible tubes, in Patel D, Vaishnav R (eds): Basic Hemodynamics and Its Role in Disease Processes. Baltimore: University Park Press, 1980, pp 419-423

35. Garg K, Tandon V, Sinha S, Suri A, Mahapatra AK, Sharma BS: Remote site intracranial hemorrhage: our experience and review of literature. Neurol India 62:296-302, 2014

36. Gisolf J, van Lieshout JJ, van Heusden K, Pott F, Stok WJ, Karemaker JM: Human cerebral venous outflow pathway depends on posture and central venous pressure. J Physiol 560:317-327, 2004

37. Grady MS, Bedford RF, Park TS: Changes in superior sagittal sinus pressure in children with head elevation, jugular venous compression, and PEEP. J Neurosurg 65:199-202, 1986

38. Grant R, Condon B, Hart I, Teasdale GM: Changes in intracranial CSF volume after lumbar puncture and their relationship to post-LP headache. J Neurol Neurosurg Psychiatry 54:440-442, 1991

39. Greenfield JC Jr, Tindall GT: Effect of acute increase in 
intracranial pressure on blood flow in the internal carotid artery of man. J Clin Invest 44:1343-1351, 1965

40. Hamilton MG, Price AV: Syndrome of inappropriately lowpressure acute hydrocephalus (SILPAH). Acta Neurochir Suppl 113:155-159, 2012

41. Higashi S, Futami K, Matsuda H, Yamashita J, Hashimoto M, Hasegawa M, et al: Effects of head elevation on intracranial hemodynamics in patients with ventriculoperitoneal shunts. J Neurosurg 81:829-836, 1994

42. Huang CY, Hung YC, Tai SH, Lee EJ: Cerebellar hemorrhage after multiple manual pumping tests of a ventriculoperitoneal shunt: a case report. Kaohsiung J Med Sci 25:29-33, 2009

43. Iwabuchi T, Sobata E, Suzuki M, Suzuki S, Yamashita M: Dural sinus pressure as related to neurosurgical positions. Neurosurgery 12:203-207, 1983

44. Johnston IH, Rowan JO: Raised intracranial pressure and cerebral blood flow. 3. Venous outflow tract pressures and vascular resistances in experimental intracranial hypertension. J Neurol Neurosurg Psychiatry 37:392-402, 1974

45. Kajimoto Y, Ohta T, Miyake H, Matsukawa M, Ogawa D, Nagao K, et al: Posture-related changes in the pressure environment of the ventriculoperitoneal shunt system. J Neurosurg 93:614-617, 2000

46. Kerr EE, Prevedello DM, Jamshidi A, Ditzel Filho LF, Otto BA: Immediate complications associated with high-flow cerebrospinal fluid egress during endoscopic endonasal skull base surgery. Neurosurg Focus 37(4):E3, 2014

47. Kety SS, Shenkin HA, Schmidt CF: The effects of increased intracranial pressure on cerebral circulatory functions in man. J Clin Invest 27:493-499, 1948

48. Knowlton FP, Starling EH: The influence of variations in temperature and blood-pressure on the performance of the isolated mammalian heart. J Physiol 44:206-219, 1912

49. Lesniak MS, Clatterbuck RE, Rigamonti D, Williams MA: Low pressure hydrocephalus and ventriculomegaly: hysteresis, non-linear dynamics, and the benefits of CSF diversion. Br J Neurosurg 16:555-561, 2002

50. Luce JM, Huseby JS, Kirk W, Butler J: A Starling resistor regulates cerebral venous outflow in dogs. J Appl Physiol 53:1496-1503, 1982

51. Magnaes B: Body position and cerebrospinal fluid pressure. Part 2: clinical studies on orthostatic pressure and the hydrostatic indifferent point. J Neurosurg 44:698-705, 1976

52. Magnaes B: Clinical studies of cranial and spinal compliance and the craniospinal flow of cerebrospinal fluid. Br J Neurosurg 3:659-668, 1989

53. Magnaes B: Movement of cerebrospinal fluid within the craniospinal space when sitting up and lying down. Surg Neurol 10:45-49, 1978

54. Marmarou A, Shulman K, LaMorgese J: Compartmental analysis of compliance and outflow resistance of the cerebrospinal fluid system. J Neurosurg 43:523-534, 1975

55. Marquardt G, Setzer M, Schick U, Seifert V: Cerebellar hemorrhage after supratentorial craniotomy. Surg Neurol 57:241-252, 2002

56. Martínez-Lage JF, Alarcón F, Alfaro R, Ruíz-Espejo A, López-Guerrero AL, Hernández-Abenza J: Cervical extramedullary mass lesion due to chronic CSF overshunting: case report and literature review. Childs Nerv Syst 25:895-898, 2009

57. Matsumoto K, Ohta M, Takeshita I: Symptomatic spinal extramedullary mass lesion secondary to chronic overdrainage of ventricular fluid-case report. Neurol Med Chir (Tokyo) 42:140-142, 2002

58. McCullough DC: Symptomatic progressive ventriculomegaly in hydrocephalics with patent shunts and antisiphon devices. Neurosurgery 19:617-621, 1986

59. Miyazaki T, Chiba A, Nishina H, Uesaka Y, Nakase H, Kanazawa I: Upper cervical myelopathy associated with low
CSF pressure: a complication of ventriculoperitoneal shunt. Neurology 50:1864-1866, 1998

60. Moayeri NN, Henson JW, Schaefer PW, Zervas NT: Spinal dural enhancement on magnetic resonance imaging associated with spontaneous intracranial hypotension. Report of three cases and review of the literature. J Neurosurg 88:912-918, 1998

61. Mokri B: The Monro-Kellie hypothesis: applications in CSF volume depletion. Neurology 56:1746-1748, 2001

62. Mokri B, Parisi JE, Scheithauer BW, Piepgras DG, Miller GM: Meningeal biopsy in intracranial hypotension: meningeal enhancement on MRI. Neurology 45:1801-1807, 1995

63. Müller LO, Toro EF: Enhanced global mathematical model for studying cerebral venous blood flow. J Biomech 47:33613372,2014

64. Nakagawa Y, Tsuru M, Yada K: Site and mechanism for compression of the venous system during experimental intracranial hypertension. J Neurosurg 41:427-434, 1974

65. Osterholm JL: Reaction of the cerebral venous sinus system to acute intracranial hypertension. J Neurosurg 32:654-659, 1970

66. Owler BK, Jacobson EE, Johnston IH: Low pressure hydrocephalus: issues of diagnosis and treatment in five cases. Br J Neurosurg 15:353-359, 2001

67. Pang D, Altschuler E: Low-pressure hydrocephalic state and viscoelastic alterations in the brain. Neurosurgery 35:643656, 1994

68. Pang Q, Wang C, Hu Y, Xu G, Zhang L, Hao X, et al: Experimental study of the morphology of cerebral bridging vein. Chin Med Sci J 16:19-22, 2001

69. Pfister R, Bussmann L, Reinhart WH: Fatal intracerebral hemorrhage after reducing the valve pressure of a ventriculoperitoneal shunt. Acta Neurochir (Wien) 151:409-410, 2009

70. Piechnik SK, Czosnyka M, Richards HK, Whitfield PC, Pickard JD: Cerebral venous blood outflow: a theoretical model based on laboratory simulation. Neurosurgery 49:12141222,2001

71. Pomeranz S, Beni L, Shalit MN: The effect of intracranial hypotension on cerebral blood flow in a feline model. Acta Neurochir (Wien) 122:113-117, 1993

72. Portnoy HD, Branch C, Castro ME: The relationship of intracranial venous pressure to hydrocephalus. Childs Nerv Syst 10:29-35, 1994

73. Qvarlander S, Sundström N, Malm J, Eklund A: Postural effects on intracranial pressure: modeling and clinical evaluation. J Appl Physiol (1985) 115:1474-1480, 2013

74. Rahme R, Weil AG, Sabbagh M, Moumdjian R, Bouthillier A, Bojanowski MW: Decompressive craniectomy is not an independent risk factor for communicating hydrocephalus in patients with increased intracranial pressure. Neurosurgery 67:675-678, 2010

75. Sassa T: The role of human-specific gene duplications during brain development and evolution. J Neurogenet 27:86-96, 2013

76. Schievink WI, Meyer FB, Atkinson JL, Mokri B: Spontaneous spinal cerebrospinal fluid leaks and intracranial hypotension. J Neurosurg 84:598-605, 1996

77. Seoane E, Rhoton AL Jr: Compression of the internal jugular vein by the transverse process of the atlas as the cause of cerebellar hemorrhage after supratentorial craniotomy. Surg Neurol 51:500-505, 1999

78. Shapiro K, Fried A, Takei F, Kohn I: Effect of the skull and dura on neural axis pressure-volume relationships and CSF hydrodynamics. J Neurosurg 63:76-81, 1985

79. Shapiro K, Marmarou A, Shulman K: Characterization of clinical CSF dynamics and neural axis compliance using the pressure-volume index: I. The normal pressure-volume index. Ann Neurol 7:508-514, 1980 
80. Si Z, Luan L, Kong D, Zhao G, Wang H, Zhang K, et al: MRI-based investigation on outflow segment of cerebral venous system under increased ICP condition. Eur J Med Res 13:121-126, 2008

81. Stiver SI: Complications of decompressive craniectomy for traumatic brain injury. Neurosurg Focus 26(6):E7, 2009

82. Tobinick E, Vega CP: The cerebrospinal venous system: anatomy, physiology, and clinical implications. MedGenMed 8:53, 2006

83. Turkoglu E, Kazanci B, Karavelioglu E, Sanli M, Kazanci B, Sekerci Z: Intracerebral hematoma following lumboperitoneal shunt insertion: a rare case report. Turk Neurosurg 21:94-96, 2011

84. Ulrich NH, Maier M, Bernays RL, Krayenbuhl N, Kollias S: Cervical myelopathy due to chronic overshunting in a pediatric patient: case report and review of the literature. Turk Neurosurg 23:410-414, 2013

85. Valdueza JM, von Münster T, Hoffman O, Schreiber S, Einhäupl KM: Postural dependency of the cerebral venous outflow. Lancet 355:200-201, 2000

86. Van Roost D, Thees C, Brenke C, Oppel F, Winkler PA, Schramm J: Pseudohypoxic brain swelling: a newly defined complication after uneventful brain surgery, probably related to suction drainage. Neurosurgery 53:1315-1327, 2003

87. Vassilyadi M, Farmer JP, Montes JL: Negative-pressure hydrocephalus. J Neurosurg 83:486-490, 1995

88. Vignes JR, Dagain A, Guérin J, Liguoro D: A hypothesis of cerebral venous system regulation based on a study of the junction between the cortical bridging veins and the superior sagittal sinus. Laboratory investigation. J Neurosurg 107:1205-1210, 2007

89. Vogels RL, Verstegen MJ, van Furth WR: Cerebellar haemorrhage after non-traumatic evacuation of supratentorial chronic subdural haematoma: report of two cases. Acta Neurochir (Wien) 148:993-996, 2006

90. Waziri A, Fusco D, Mayer SA, McKhann GM II, Connolly ES Jr: Postoperative hydrocephalus in patients undergoing decompressive hemicraniectomy for ischemic or hemorrhagic stroke. Neurosurgery 61:489-494, 2007

91. Wolfe SQ, Bhatia S, Green B, Ragheb J: Engorged epidural venous plexus and cervical myelopathy due to cerebrospinal fluid overdrainage: a rare complication of ventricular shunts. Case report. J Neurosurg 106 (3 Suppl):227-231, 2007

92. Wood B: Human evolution. BioEssays 18:945-954, 1996

93. Yaşargil MG, Yonekawa Y: Results of microsurgical extraintracranial arterial bypass in the treatment of cerebral ischemia. Neurosurgery 1:22-24, 1977

94. Zamboni P, Sisini F, Menegatti E, Taibi A, Malagoni AM, Morovic S, et al: An ultrasound model to calculate the brain blood outflow through collateral vessels: a pilot study. BMC Neurol 13:81, 2013

95. Zippel KC, Lillywhite HB, Mladinich CR: New vascular system in reptiles: anatomy and postural hemodynamics of the vertebral venous plexus in snakes. J Morphol 250:173-184, 2001

\section{Disclosures}

The author reports no conflict of interest concerning the materials or methods used in this study or the findings specified in this paper.

\section{Correspondence}

Kaveh Barami, Kaiser Permanente, 2025 Morse Ave., Sacramento, CA 95825. email:kbarami@yahoo.com. 